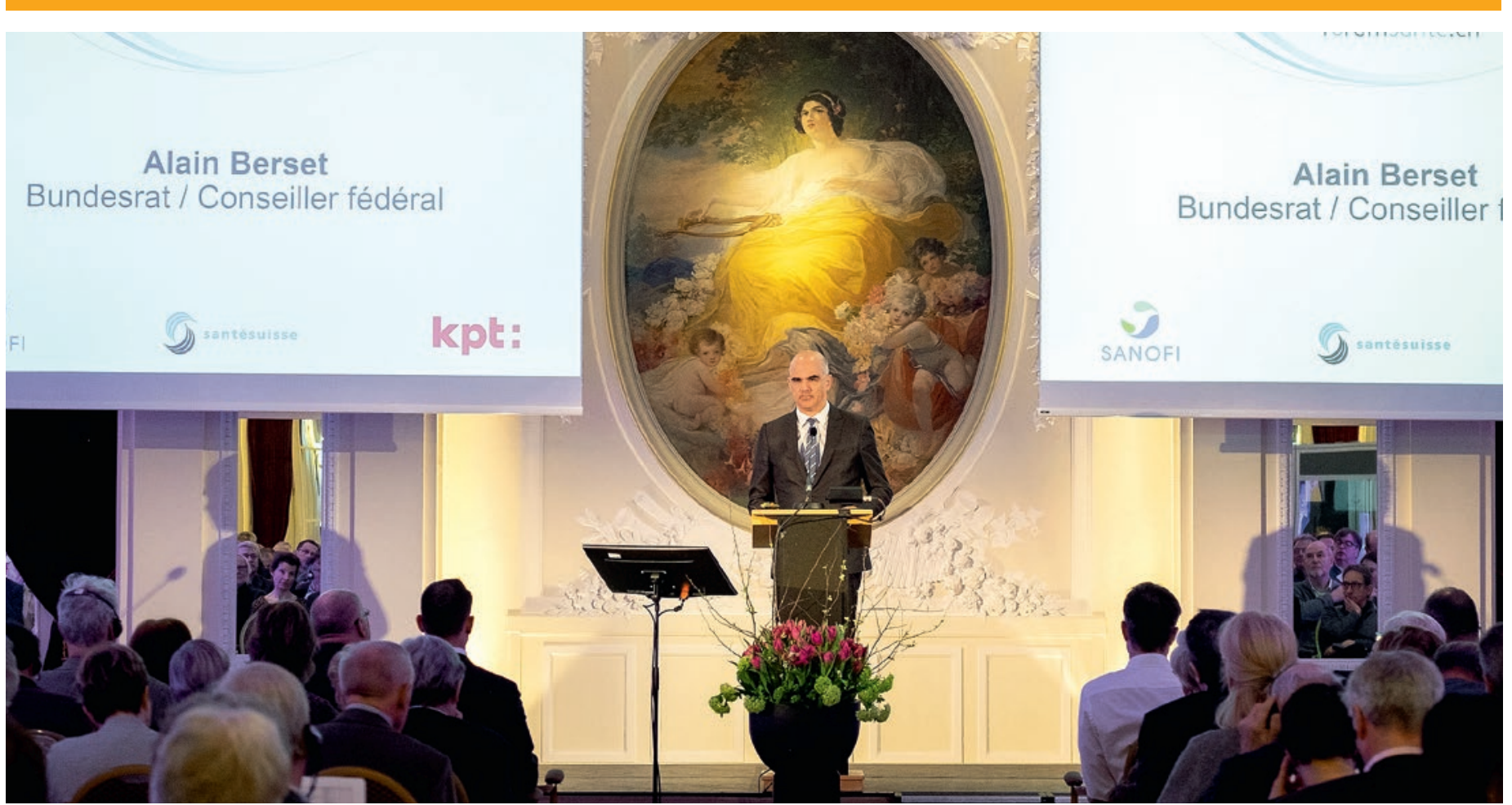

Le conseiller fédéral Alain Berset a donné le coup d'envoi du $21^{\mathrm{e}}$ forumsante.ch.

\title{
Profession recherche nouvelle image de soi
}

\section{Daniel Lüthi}

Journaliste indépendant et photographe, conseiller médiatique, Berne

Les temps changent - et le corps médical essaie de s'adapter. Comment la conception du rôle de médecin évolue-t-elle? Quelle sera l'image de la profession à l'avenir? Telles étaient les questions posées lors du dernier congrès "forumsanté» à Berne. L'annonce finale de l'arrêt de la manifestation sous sa forme actuelle a suscité des regrets mais n'a pas vraiment surpris.

La pression économique, ou «folie économique» comme l'appelle Bertrand Kiefer, rédacteur en chef de la Revue Médicale Suisse, a fréquemment surgi dans le débat à l'hôtel Bellevue. Ironie du sort: la manifestation, qui a régulièrement servi de plateforme prestigieuse à des thèmes centraux de ce genre au cours des vingt dernières années, est à son tour victime du pouvoir de l'argent. En s'obligeant à respecter les règles éthiques en vigueur - $\mathrm{y}$ compris les propres règles de «forumsanté» - Jacques de Haller, président de forumsante.ch, a admis ne plus avoir réussi à récolter suffi- samment de fonds auprès des sponsors. «Ce feu d'artifice était donc aussi un bouquet final.»

\section{Travailler ensemble, et non en parallèle}

C'est le ministre de la santé en personne qui a ouvert le bal d'intervenants de renom, et Alain Berset a cité deux éléments clés qui expliquent l'évolution fulgurante de l'image de la profession de médecin: la numérisation et l'évolution démographique. "La marche du temps ne peut être stoppée», a philosophé un conseiller fédéral 
très en verve, il s'agit de l'«accompagner intelligemment sur le plan stratégique». Sur le plan politique, les cantons sont en première ligne: «Nous formons bien trop peu de médecins en Suisse.»

En matière de numérisation, les compétences des médecins sont encore trop lacunaires, comme le montre une étude de l'OCDE. Mais il faut aussi revoir la répartition des tâches dans le secteur de la santé et renforcer la coordination.

Un avis partagé par Daniel Scheidegger, président de l'Académie suisse des sciences médicales (ASSM). "Nous ne travaillons pas ensemble mais en parallèle», a-t-il affirmé en référence aux différentes disciplines du secteur de la santé. Et il s'est posé la question suivante: «Avons-nous trop peu de personnel - ou pratiquons-nous trop d'actes médicaux?» Sa réponse: «De nombreux actes pratiqués sont inutiles. Le patient a aussi le droit de ne pas savoir.» Dans son exposé à la fois engagé et décalé, Daniel Scheidegger a par ailleurs fait le lien avec le débat actuel sur le climat et esquissé la vision d'une médecine durable - une médecine avec moins de gaspillage, moins de matériel à usage unique, moins de voyages en avion. Sa question rhétorique à ce propos: «Le secteur de la santé est-il un programme d'encouragement de l'économie?»

\section{Le médecin en tant qu'entrepreneur?}

Et voilà à nouveau évoqué le rôle de l'économie. "La colonisation de nos univers de vie par l'économie est un fait incontestable», a déclaré Frank Ulrich Montgomery, président du Conseil de l'Association médicale

\section{Et voilà à nouveau évoqué le rôle de l'économie.}

mondiale et président du Comité permanent des médecins européens (CPME). C'est pourquoi, contrairement au président de l'ASSM Daniel Scheidegger, il est favorable à ce que les médecins suivent également un MBA afin de manifester leur intérêt pour les aspects

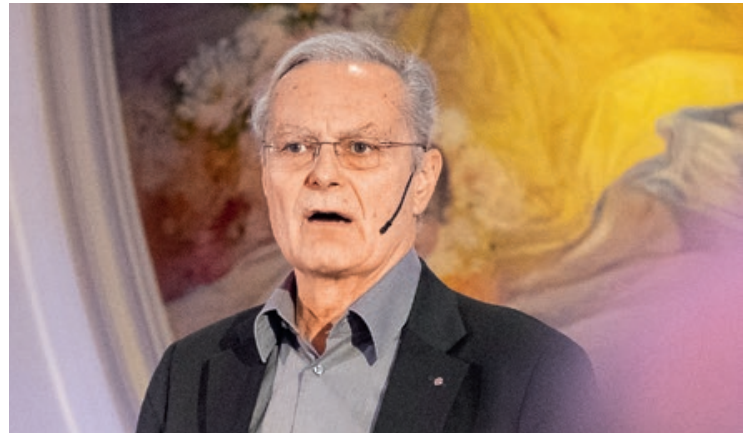

Daniel Scheidegger, président de l'Académie suisse des sciences médicales (ASSM)

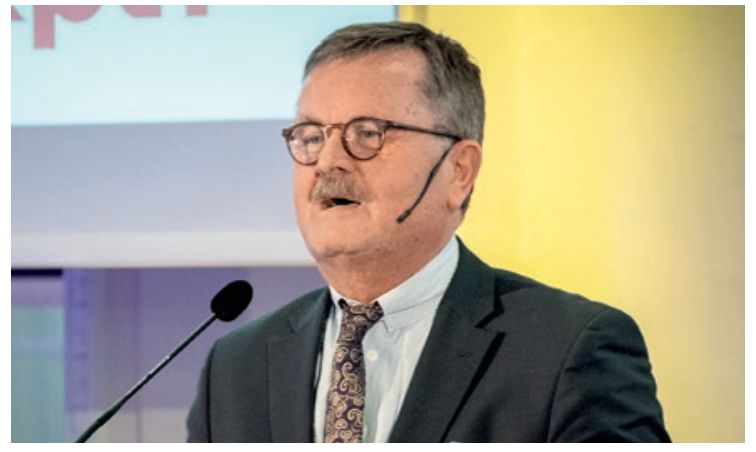

Frank Ulrich Montgomery, président du Conseil de l'Association médicale mondiale et président du Comité permanent des médecins européens (CPME)

économiques de la santé - «économiques, et pas mercantiles», a-t-il tenu à préciser. "Nous ne devons pas devenir des entrepreneurs répondant à des incitations sous forme de bonus.»

Frank Ulrich Montgomery a parfaitement reflété l'esprit du congrès: des hommes et une femme reconnus

\section{Pratiquement tous les intervenants ont parlé de l'image de soi du médecin.}

dans la profession ont comparé le monde actuel de la médecine à celui de leurs débuts et cherché à en tirer des enseignements pour le futur. "Nous étions des demi-dieux en blouse blanche, nous ne le sommes plus», constate Frank Ulrich Montgomery. En tant que médecin à l'âge de la retraite, il se déclare optimiste vis-à-vis de la jeune génération de médecins: «Ils ont raison d'exiger une plus grande autonomie et un meilleur équilibre entre vie professionnelle et vie privée.» Pratiquement tous les intervenants ont parlé de l'image de soi du médecin, notamment Jacques de Haller dans son introduction: «Notre position au sein de la société et nos prérogatives sont remises en cause - et nous sommes sur la défensive.» Il convient de se trouver une nouvelle identité positive, de redéfinir le rôle, l'identité du docteur. Jacques de Haller parle à ce propos du médecin comme un "coach santé».

Le président de l'ASSM Daniel Scheidegger a déclaré sans fard: "Le temps des grands pontes est révolu.» Il y a certaines choses que d'autres font aussi bien, sinon mieux, qu'un médecin. La photo illustrant cette affirmation était celle d'un robot effectuant une injection.

\section{L'homme et la machine}

Un autre thème clé abordé lors du dernier forumsanté était le rôle de la technique, de l'informatique, le pouvoir des données numériques et la question de savoir si 


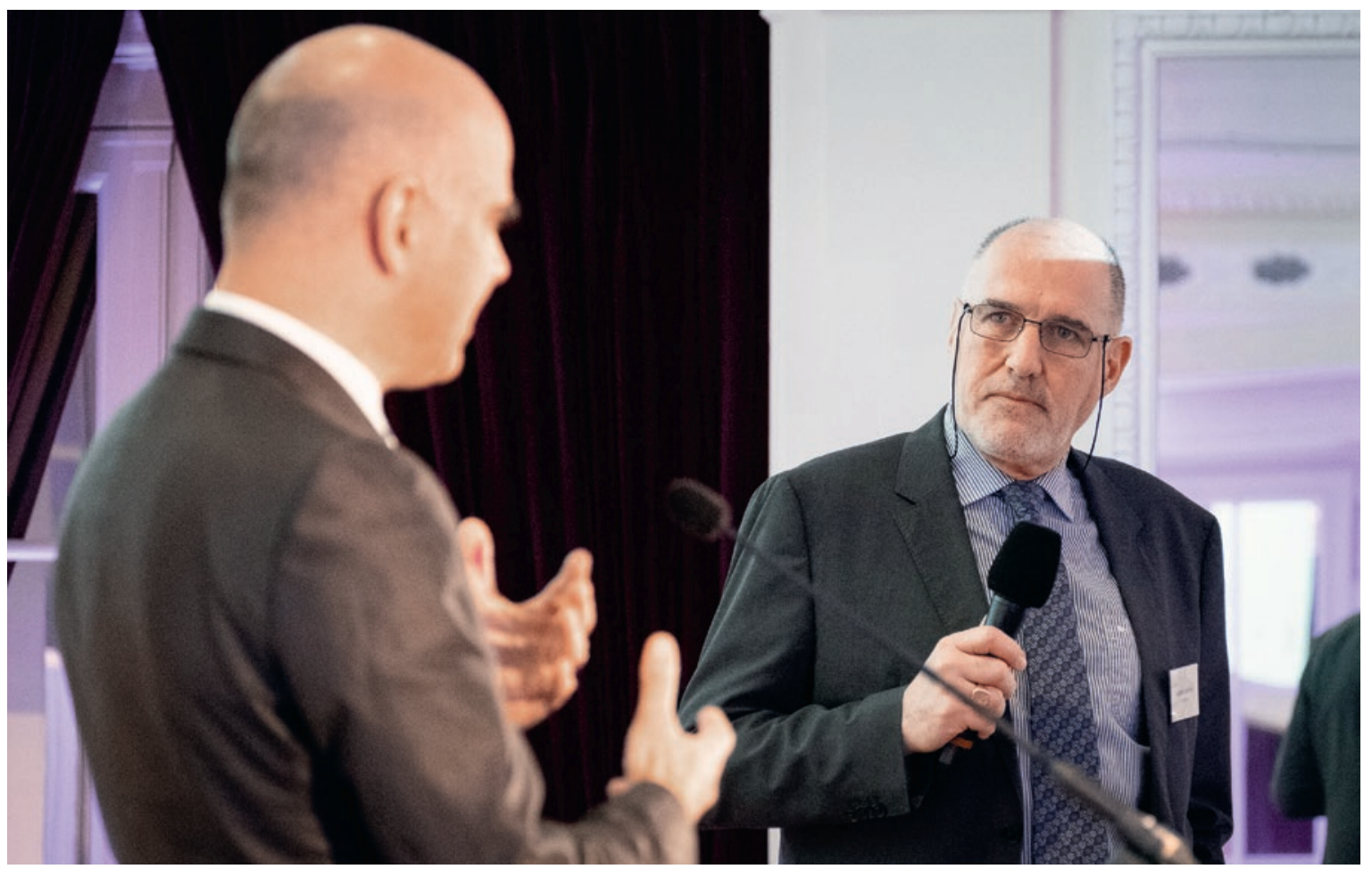

Le ministre de la Santé Alain Berset en conversation avec Jacques de Haller, président de forumsante.ch.

les machines finiront un jour par remplacer l'humain. A ce sujet, Bertrand Kiefer, médecin, théologien et journaliste, affirme: «Ecouter et servir d'intermédiaire, un robot en est incapable.» Aborder l'aspect humain, la souffrance, la peur de la mort, seul un humain peut le

\section{Les machines remplaceront-elles un jour les humains? Cette question était également un des thèmes phares abordés.}

faire - un médecin par exemple. «Les hôpitaux sont les églises d'aujourd'hui», estime Bertrand Kiefer, car le soin n'est pas un produit quantifiable.

Christian Lovis, directeur du service des sciences de l'information médicale à l'Université de Genève, a évoqué quant à lui de façon percutante et très parlante l'intelligence artificielle et le big data. Il a passé de la musique qui aurait pu être du Bach ou du Beethoven mais avait été intégralement produite et jouée par des machines, et s'est enthousiasmé de ces nouvelles possibilités techniques. En ce qui concerne la médecine et le thème du congrès, à savoir le futur rôle des médecins, il s'est montré bien plus nuancé et prudent. Il affirme que l'intelligence artificielle en médecine est comme un test de grossesse: un objet dont la fiabilité est discutable et dont les résultats doivent être pondérés et interprétés par quelqu'un disposant des compétences nécessaires.

Sa conclusion est apparue comme une lueur d'espoir: "Tant qu'il y aura des hommes et des femmes, nous aurons besoin de médecins.»

\section{Crédits photo}

Sascha Hähni

\section{L'essentiel en bref}

- La $21^{\mathrm{e}}$ édition de forumsante.ch a eu lieu le 14 janvier à Berne, à I'Hôtel Bellevue.

- $\quad C^{\prime}$ est le Conseiller fédéral et ministre de la santé Alain Berset qui a ouvert la journée, comme premier orateur.

- Il a été question du changement de l'image professionnelle des médecins, dans un temps de digitalisation et d'évolution démographique; de la vision d'une médecine durable face au changement climatique; ou encore du rôle de l'économie.

- Le congrès n'aura plus lieu l'année prochaine, faute d'avoi pu assurer un financement suffisant tout en respectant les règles éthiques en vigueur, y compris celles propres à forumsanté. 